\title{
CDISC SEND Within Normal Limits Results Terminology
}

National Cancer Institute

\section{Source}

National Cancer Institute. CDISC SEND Within Normal Limits Results Terminology. NCI

Thesaurus. Code C132321.

Terminology associated with the within normal limits results codelist of the Clinical Data Interchange Standards Consortium (CDISC) Standard for the Exchange of Non-clinical Data (SEND). 